Zhang Qi

Wen Guang $\bowtie$

Luo Lan

Tang Rui

https://doi.org/10.21278/TOF.444011819

ISSN 1333-1124

eISSN 1849-1391

\title{
CONTACT STRESS PREDICTION MODEL FOR VARIABLE HYPERBOLIC CIRCULAR ARC GEAR BASED ON THE OPTIMIZED KRIGING-RESPONSE SURFACE MODEL
}

\begin{abstract}
Summary
In order to study the influence of design parameters (pressure angle, tooth width, tooth line radius, modulus, and moment) on contact stress of variable hyperbolic circular arc gear (VHCAG) and to obtain the best manufacturing parameters, The Kriging-Response Surface Model, a hybrid surrogate model with adaptive quantum particle swarm optimization (QPSO) algorithm was proposed to establish the expression prediction model for the relation between design parameters and contact stress. An intelligent quantum particle swarm optimization algorithm based on adaptive weight and natural selection is proposed to optimize the parameters of Gaussian variation function of the kriging surrogate model to improve its fitting accuracy. The global search ability of quantum particles is improved, and the accuracy and stability of the algorithm are improved by adjusting the weight of quantum particles adaptively and by optimizing the elimination iteration process, and the response relationship between design parameters and contact stress was established. The binomial response surface model of gear design parameters and contact stress is established based on the output obtained through the improved kriging model; this simplifies the complex expression of the kriging model. The effects of parameters and their cross-terms on contact stress are analysed based on the contact stress prediction model established by using the optimized Kriging-Response Surface Model hybrid surrogate model. The hybrid Kriging-Response Surface Model surrogate model lays a foundation for the research on the reliability and robust optimization of cylindrical gears with variable hyperbolic arc tooth profile.
\end{abstract}

Key words: $\quad$ contact stress, design parameters, Latin square sampling method, prediction model, hybrid surrogate model, parametric impact analysis

\section{Introduction}

Variable hyperbolic circular arc gear (VHCAG) is a new type of transmission devices, which, theoretically, has the characteristics of high contact rate, high bearing capacity, no axial force, high coincidence, high transmission efficiency, long service life, high stability, and low noise [1-6]. However, the research on this kind of gear is still in its infancy; the gear performance has not been verified, so its industrial promotion is limited. The relationship 
between design parameters and contact stress is the basis of reliability analysis and robust optimization. Because of the complexity of the tooth surface of cylindrical gear with variable hyperbolic arc tooth line, it is difficult to derive the explicit formula of contact stress.

In practical engineering, the relationship between uncertainties and design objectives is complex and highly non-linear, and it is often expressed by implicit functions. A surrogate model can approximate the relationship between uncertainties and design objectives very well, and it can establish its explicit expression [7]. Cremanns et al. developed a multiobjective robust optimization method for the steam turbine labyrinth seal based on a surrogate model; the authors also solved the problem of conflict between minimizing mass flow and total enthalpy, thus improving the performance and lowering the temperature [8]. Wang et al. used a kriging surrogate model and a $6 \sigma$ robust design method to optimize the robust design of flexible mechanisms with flexible plates [9]. Wang Jie et al. used a radial basis function for the interpolation of the residual error of the response surface model in order to improve and optimize it [10]. Zhou Xiaojian proposed a surrogate model of support vector regression machine based on non-semi-positive deterministic kernels, optimized its fitting accuracy, and provided an accurate surrogate model for robust optimization design [11].

The accurate response relationship can be established and the contact stress calculation of VHCAG can be realized by using a kriging surrogate model. However, the calculation of a large number of parameters in the kriging surrogate model has become a complex optimization problem, and the calculation process is cumbersome. At the same time, the kriging surrogate model cannot get an explicit polynomial expression. Therefore, the Kriging -Response Surface model, a hybrid surrogate model with an intelligent algorithm of quantum particle swarm optimization (abbreviated as AQPSO-kriging-RSM) based on adaptive weight and natural selection is proposed to establish a binomial response surface model of the relationship between design parameters and contact stress by using the improved quantum particle algorithm. The degree of the influence of design parameters and their intersections on contact stress is analysed. Using the contact stress prediction model based on the AQPSOkriging-RSM method under the set working conditions, we can also get the best manufacturing parameter

\section{Experimental Design}

Suppose that the design parameters (tooth width, pressure angle, modulus, tooth line radius) and the torque of cylindrical gears with variable hyperbolic arc tooth line obey normal distribution. According to references [12] and [13], the mean and standard deviations of parameters are determined. When there is no test data, the standard deviation $\sigma$ is determined by the coefficient of variation method, and the coefficient of variation is 0.1 . Gear design parameters and their mean and standard deviation values are shown in Table 1.

Table 1 Average and standard deviation of design parameters of VHCAG

\begin{tabular}{|c|c|c|c|c|}
\hline Variable & Mean Value & Standard Deviation & Upper Bound & Lower Bound \\
\hline Tooth Width $(\mathrm{mm})$ & 40 & 0.5 & 38.5 & 41.5 \\
\hline Modulus $(\mathrm{mm})$ & 3.2 & 0.1 & 2.9 & 3.5 \\
\hline Pressure Angle $\left({ }^{\circ}\right)$ & 20 & 0.5 & 18.5 & 21.5 \\
\hline Radius of Tooth Line $(\mathrm{mm})$ & 250 & 25 & 175 & 325 \\
\hline Moment $(\mathrm{N} \cdot \mathrm{mm})$ & 161400 & 16140 & 112980 & 209820 \\
\hline
\end{tabular}


Considering the non-linearity of contact characteristics of cylindrical gears with variable hyperbolic arc tooth line, three levels, $k_{1}, k_{2}, k_{3}$, are selected for each design parameter in this paper, according to [13]. The values are $k_{1}=0.05, k_{2}=0.5, k_{3}=0.95$, as shown in Table 2 .

Table 2 Level of design parameters

\begin{tabular}{|c|c|c|c|}
\hline Variable & Level 1 & Level 2 & Level 3 \\
\hline Tooth Width $(\mathrm{mm})$ & 38.83685 & 40.00000 & 41.16315 \\
\hline Modulus $(\mathrm{mm})$ & 2.96737 & 3.20000 & 3.43263 \\
\hline Pressure Angle $\left({ }^{\circ}\right)$ & 18.83685 & 20.00000 & 21.16315 \\
\hline Radius of Tooth Line $(\mathrm{mm})$ & 191.84250 & 250.00000 & 308.15750 \\
\hline Moment $(\mathrm{N} \cdot \mathrm{mm})$ & 123853.51800 & 161400.00000 & 198946.48200 \\
\hline
\end{tabular}

Considering that the range of the interval is too large, the accuracy of the surrogate model will be reduced, according to [12]; thus, the point which has the greatest impact on the reliability index of this new type of gear will be excluded from the sample space and the calculation accuracy will be affected. In order to ensure the uniformity of the sample space, the optimal Latin hyper design method was used and the results were compared with those of the uniform design test. Optimization of the Latin hypercube process, which consists of inner and outer loops, is shown in Figure 1.

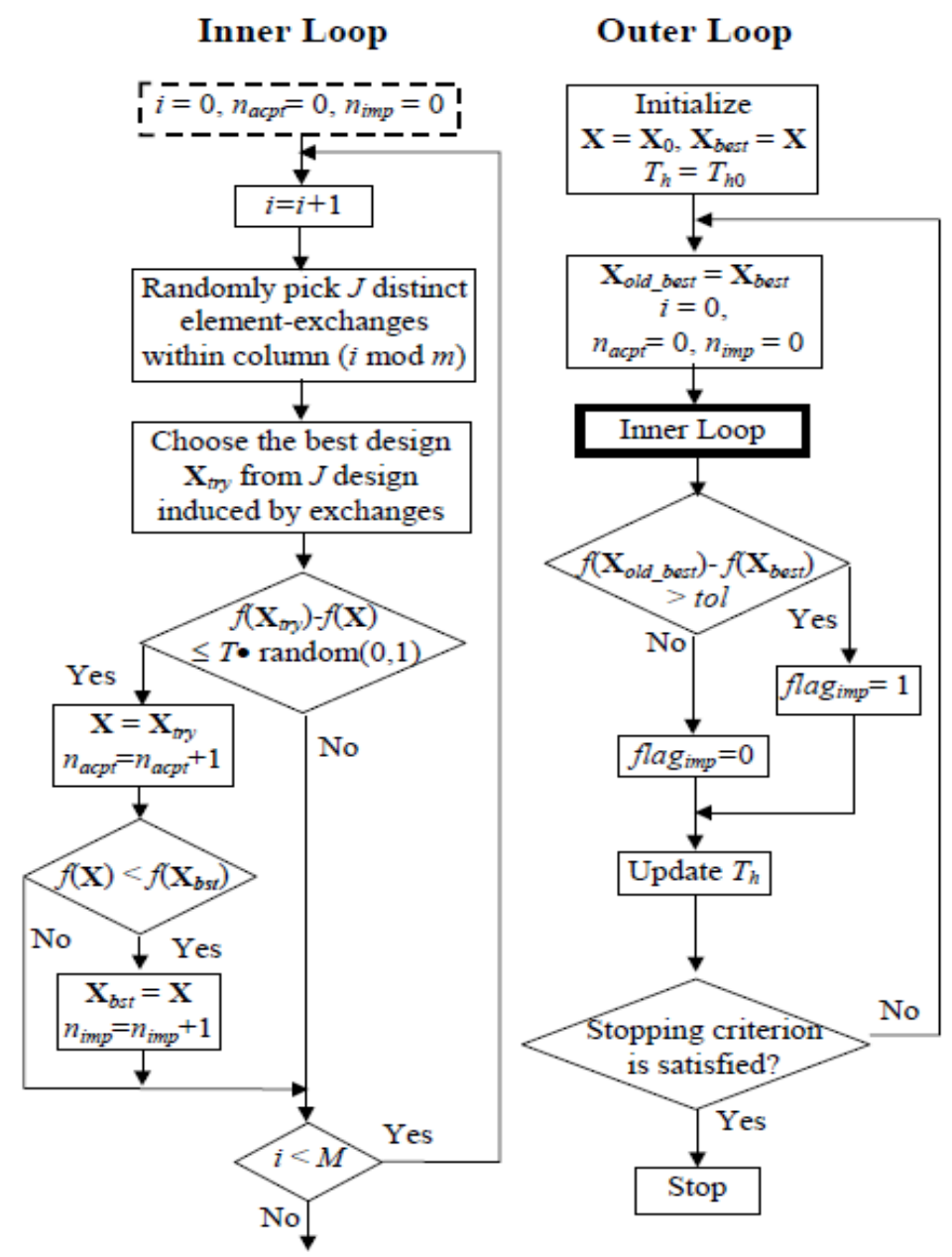

Fig. 1 Flow chart of optimized Latin hypercube 
Based on the experimental design results, the contact stress response was analysed by finite element analysis method. The analysis results are shown in Table 3.

Table 3 Contact stress response results of sample set

\begin{tabular}{|c|c|c|c|c|c|c|c|c|}
\hline No. & $\begin{array}{c}\text { Tooth } \\
\text { Number } \\
\text { of Gear }\end{array}$ & $\begin{array}{c}\text { Tooth } \\
\text { Number } \\
\text { of } \\
\text { Pinion }\end{array}$ & $\begin{array}{c}\text { Pressure } \\
\text { Angle }\end{array}$ & Modulus & $\begin{array}{c}\text { Tooth } \\
\text { Width }\end{array}$ & $\begin{array}{c}\text { Radius of } \\
\text { Tooth } \\
\text { Line }\end{array}$ & $\begin{array}{c}\text { Mome } \\
\text { nt }\end{array}$ & $\begin{array}{c}\text { Contact } \\
\text { Stress }\end{array}$ \\
\hline 1 & 31 & 62 & 1 & 3 & 2 & 3 & 1 & 384.7 \\
\hline 2 & 31 & 62 & 3 & 2 & 2 & 3 & 2 & 424.5 \\
\hline 3 & 31 & 62 & 2 & 3 & 3 & 3 & 3 & 437.4 \\
\hline 4 & 31 & 62 & 3 & 1 & 1 & 2 & 2 & 470.3 \\
\hline 5 & 31 & 62 & 2 & 1 & 1 & 2 & 3 & 506.4 \\
\hline 6 & 31 & 62 & 1 & 2 & 3 & 3 & 3 & 468.2 \\
\hline 7 & 31 & 62 & 1 & 3 & 1 & 3 & 3 & 446.6 \\
\hline$\ldots$ & $\ldots$ & $\ldots$ & $\ldots$ & $\ldots$ & $\ldots$ & $\ldots$ & $\ldots$ & $\ldots$ \\
\hline
\end{tabular}

\section{Kriging method based on the improved QPSO algorithm}

The kriging has been widely used in prediction analysis. In order to improve its accuracy and provide a more accurate mathematical model for prediction analysis, this paper proposes to optimize the kriging model using an improved quantum particle swarm optimization algorithm.

\subsection{Improved quantum particle swarm optimization}

The PSO algorithm is a heuristic intelligent algorithm. In the evolution process, the fitness function is used to evaluate the current flying position of particles, and the optimal position $p_{\text {best }}$ solution of particles is recorded in the iteration process. The global optimal position solution of the whole particle $g_{\text {best }}$ is recorded at the same time. The iteration velocity of particles in the flight process can be dynamically adjusted based on $p_{\text {best }}$ and $g_{\text {best }}$ to determine the next flight position. From the solution of Schrodinger equation, the positionupdating equation of particle swarm optimization with quantum behavior is expressed as shown in Equation 1.

$$
X_{i, j}(t+1)=p_{i, j}(t) \pm \frac{L_{i, j}(t)}{2} \ln \left(1 / u_{i, j}(t)\right), u_{i, j}(t) \sim U(0,1)
$$

The location update expression of QPSO is shown in Eq.1, where $\alpha$ is the dilationcontraction factor, $\boldsymbol{X}_{i, j}(t)$ is the position of the $i_{\text {th }}$ particle in the $j_{\text {th }}$ coordinate dimension, $\boldsymbol{p}_{i, j}(t)$ is the historically optimal location of the $i$ th particle in the $j_{\text {th }}$ coordinate dimension.

In order to further improve the convergence of QPSO algorithm, take another form of $\boldsymbol{L}_{i, j}(t)$ :

$$
\begin{aligned}
& L_{i, j}(t)=2 \alpha\left|p_{i, j}(t)-X_{i, j}(t)\right| \\
& L_{i, j}(t)=2 \alpha\left|C_{j}(t)-X_{i, j}(t)\right|
\end{aligned}
$$

Then Eq. 1 can be rewritten as:

$$
X_{i, j}(t+1)=p_{i, j}(t) \pm \alpha\left|p_{i, j}(t)-X_{i, j}(t)\right| \ln \left(1 / u_{i, j}(t)\right), u_{i, j}(t) \sim U(0,1)
$$




$$
\begin{aligned}
X_{i, j}(t & +1)=p_{i, j}(t) \pm \alpha\left|C_{j}(t)-X_{i, j}(t)\right| \ln \left(1 / u_{i, j}(t)\right), u_{i, j}(t) \sim U(0,1) \\
C_{j}(t) & =\left(C_{1}(t), C_{2}(t), \cdots, C_{N}(t)\right) \\
& =\frac{1}{M} \sum_{i=1}^{M} P_{i}(t)=\left(\frac{1}{M} \sum_{i=1}^{M} P_{i, 1}(t), \frac{1}{M} \sum_{i=1}^{M} P_{i, 2}(t), \cdots, \frac{1}{M} \sum_{i=1}^{M} P_{i, N}(t)\right)
\end{aligned}
$$

The QPSO equation can be converted, as shown in Equation 4 (Equation 5).

Inertial weight is an important parameter that can be adjusted by the QPSO algorithm. Its value has an important influence on its search ability. In the QPSO process, inertial weight decreases with the iteration algebra increase, which leads to premature local optimization and slow convergence. Based on this, an adaptive method is used to dynamically adjust the parameters so as to solve the problem of premature local optimization. At the same time, natural selection is adopted to maintain the diversity of the particle population, improve the global search ability, speed up the convergence, and improve the efficiency of the algorithm.

Definition: Individual particle evolution rate is

$$
i p_{i}(t)=\frac{\operatorname{Fitness}\left(P_{g, j}(t)\right)}{\operatorname{Fitness}\left(P_{i, j}(t)\right)}
$$

Definition: Group dispersion is

$$
\begin{aligned}
& g s_{t}(t)=\left\{g_{i, 1}(t), g_{i, 2}(t), \cdots, g_{i, N}(t)\right\} \\
& =\left\{\frac{\partial\left(p_{i, 1}(t)\right)}{\partial_{X 1}\left(X_{i, 1}(t)\right)}, \frac{\partial\left(p_{i, 2}(t)\right)}{\partial_{X 2}\left(X_{i, 2}(t)\right)}, \cdots, \frac{\partial\left(p_{i, N}(t)\right)}{\partial_{X N}\left(X_{i, N}(t)\right)}\right\}
\end{aligned}
$$

Then the equation of the improved QPSO algorithm is

$$
\begin{gathered}
p_{i, j}(t)=i p_{i}(t) P_{g, j}(t)+\varphi_{i, j}(t) i p_{i}(t)\left[P_{i, j}(t)-P_{g, j}(t)\right] \\
C_{j}(t)=\left(\frac{1}{M} \sum_{i=1}^{M} P_{i, 1}(t), \frac{1}{M} \sum_{i=1}^{M} P_{i, 2}(t), \cdots, \frac{1}{M} \sum_{i=1}^{M} P_{i, N}(t)\right) \\
X_{i, j}(t+1)=p_{i, j}(t) \pm \alpha\left|C_{j}(t)-\left(1-g s_{i, j}(t)\right) X_{i, j}(t)\right| \ln \left(1 / u_{i, j}(t)\right), u_{i, j}(t) \sim U(0,1)
\end{gathered}
$$

Adaptive evolution speed and dynamic population dispersion are introduced in Equations 9 and 11 respectively. Adaptive evolution speed is a dynamic parameter between 0 1 , which represents the proximity between an individual optimum and the global optimum. When it approaches 0 positively, it indicates that there is a great difference between the global optimum and the individual optimum, and the evolution speed is fast; otherwise, it indicates that the difference between the global optimum and the individual optimum can be accepted and the evolution speed is slower. By introducing the adaptive evolution speed, the convergence speed of the algorithm is improved. The dynamic population dispersion is used to characterize the diversity and stability of its particles. The dynamic dispersion tends to be 1 in the iteration process. The diversity of its particles is guaranteed by adaptive population dispersion. 
Q. Zhang, G. Wen,

L. Luo, R. Tang
Contact Stress Prediction Model for Variable Hyperbolic Circular Arc Gear Based on the Optimized Kriging-Response Surface Model

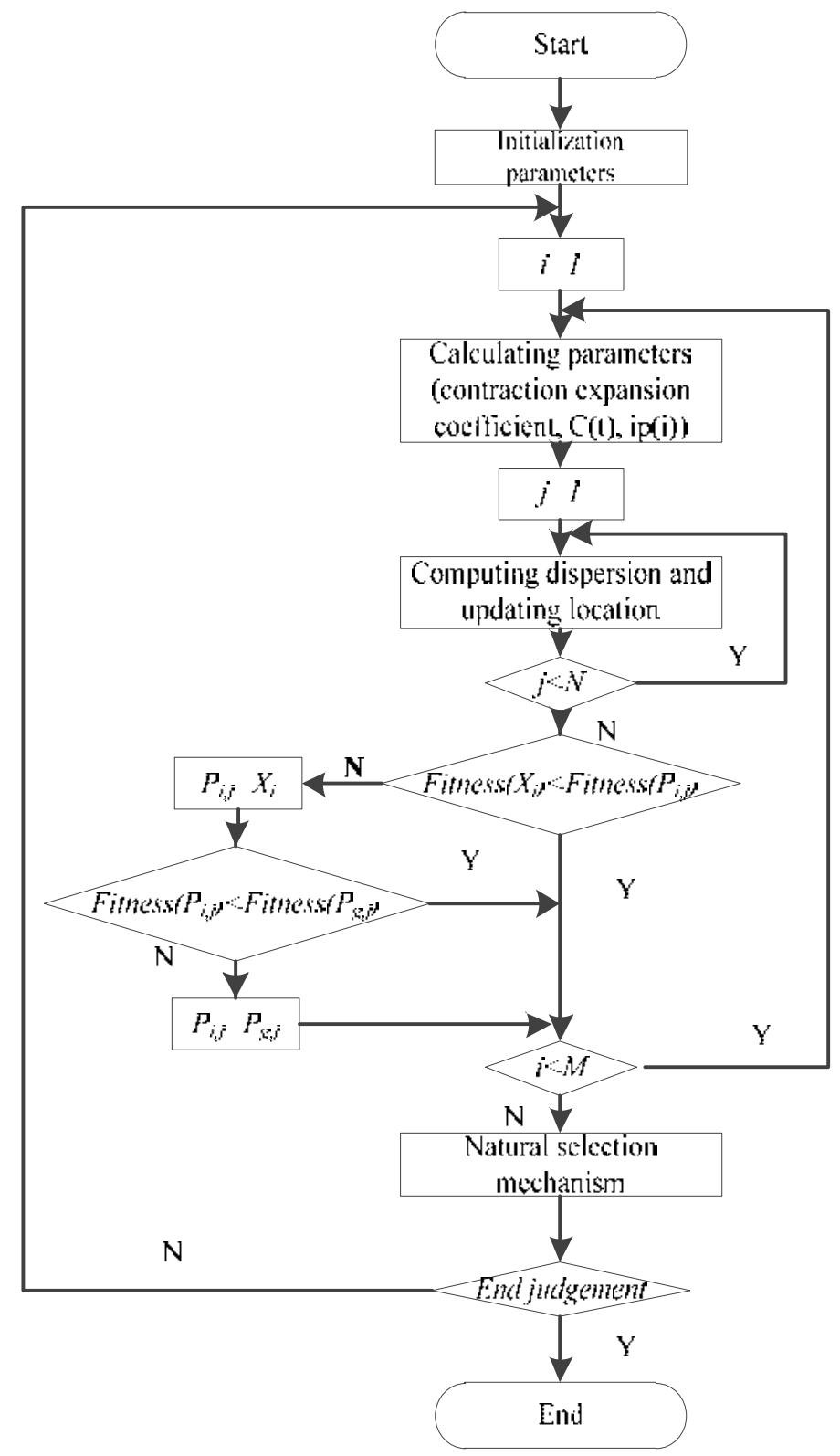

Fig. 2 Flowchart of improved QPSO algorithm

At the same time, the natural selection mechanism is used to eliminate the worst particles in the iteration process to improve the diversity of particles; thus the accuracy and stability of the algorithm are improved. The flowchart of the improved QPSO algorithm is shown in Figure 2.

In order to verify the effectiveness of the proposed algorithm, the single-peak sphere function and the multi-peak Rastrigrin function are selected to test and verify the proposed algorithm. The specific expressions of the sphere and the Rastrigrin function are referred to in the relevant literature. In this paper, no specific explanations are given. The QPSO and the improved QPSO algorithm are used to optimize the sphere the convergence curve is shown in Figure 3. 


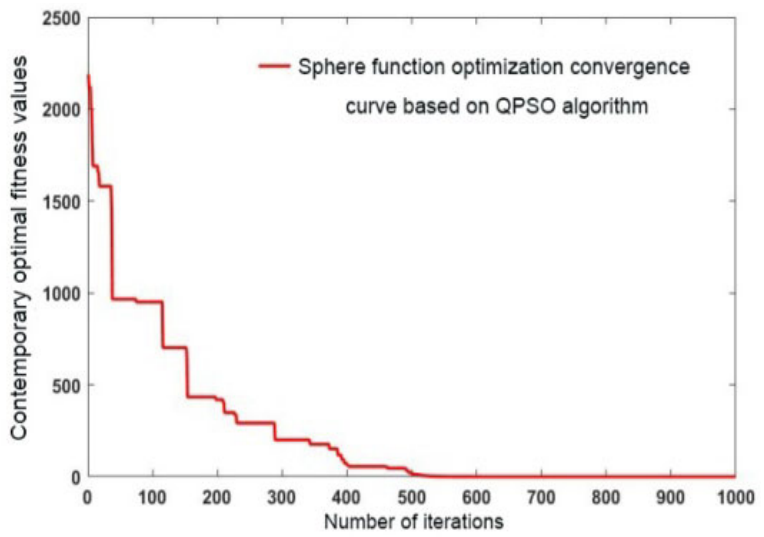

(a) QPSO algorithm

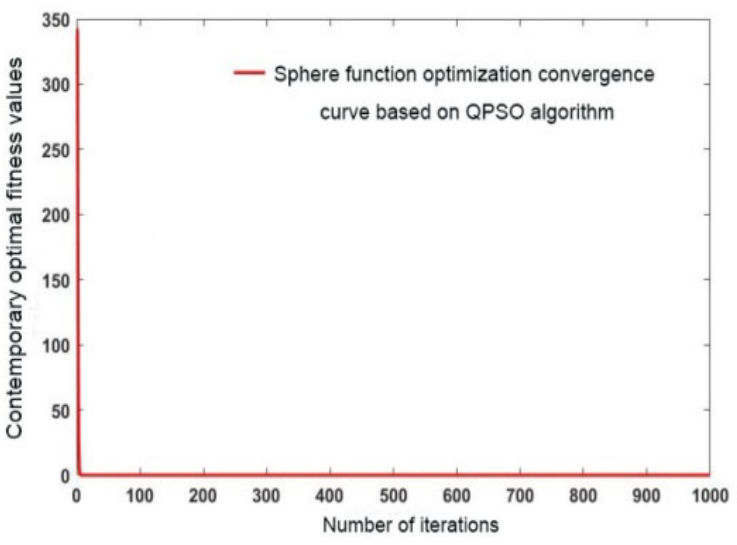

(b) Improved QPSO algorithm

Fig. 3 Optimal convergence curve of the sphere function

From Figure 3, we can see that the improved QPSO algorithm proposed in this paper can achieve the global optimization of the sphere function and that the convergence speed is significantly faster in the case of the improved QPSO algorithm than in that of the QPSO algorithm.

The QPSO and improved QPSO algorithms are used to optimize the Rastrigrin function. The convergence curve of the Rastrigrin function is shown in Figure 4.

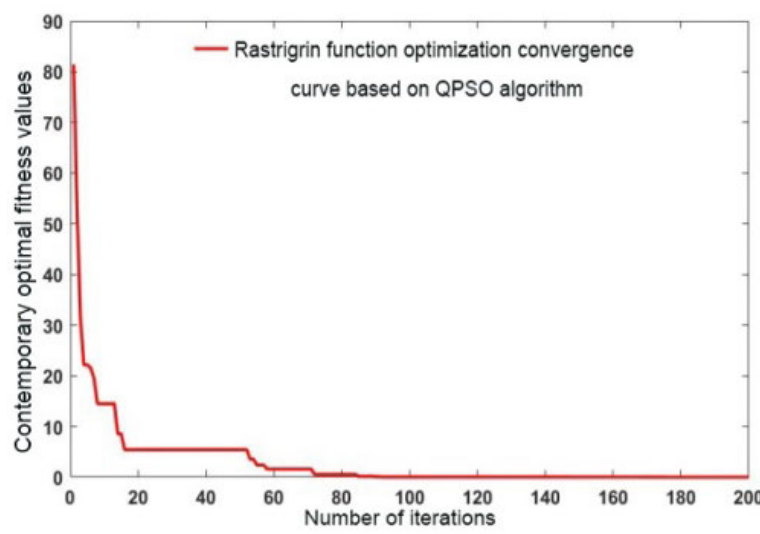

(a) QPSO algorithm

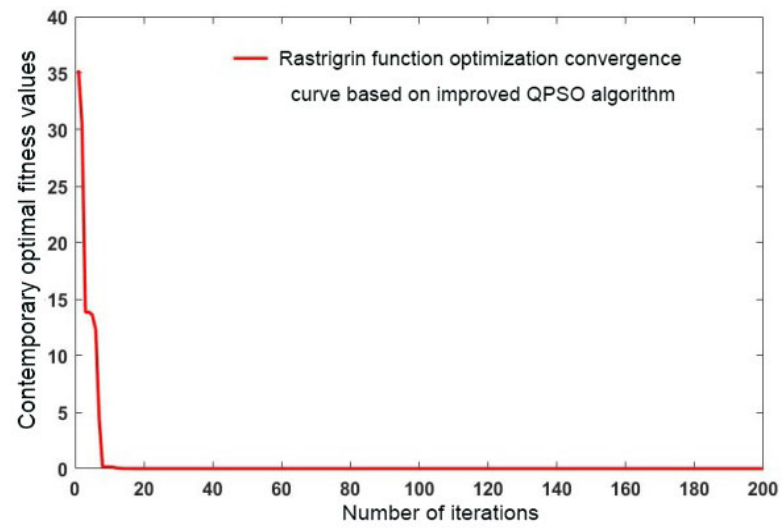

(b) Improved QPSO algorithm

Fig. 4 Optimal convergence curve of the Rastrigrin function

From Figure 4, we can see that the improved QPSO algorithm proposed in this paper can achieve the global optimization of Rastrigrin function, and that the convergence speed is significantly faster in the case of the improved QPSO algorithm than in that of the QPSO algorithm.

\subsection{Kriging surrogate model optimization based on the improved QPSO}

The kriging surrogate model is constructed by using the statistical method based on a stochastic process and by minimizing the variance of the prediction model. The model has the ability of local estimation. Even when dealing with strong non-linear problems, the model can still obtain better prediction results. It has been widely used in the field of engineering optimization.

The kriging model expresses the relationship between the input variables and response values as follows

$$
y(x)=F(\beta, x)+z(x)=f(x) \beta+z(x)
$$


Here, $f(x)$ is the deterministic part approaching the input space globally, usually in the form of polynomials; $\beta$ is the regression coefficient; $z(x)$ is the stationary Gauss process, which is used to generate local deviations. It has the following statistical characteristics

$$
\begin{aligned}
& E[z(x)]=0 \\
& \operatorname{Var}[z(x)]=\sigma^{2} \\
& E\left[z\left(x^{(i)}\right), z\left(x^{(j)}\right)\right]=\sigma^{2} R\left(\theta, x^{(i)}, x^{(j)}\right)
\end{aligned}
$$

Among them, $R\left(\theta, x^{(i)}, x^{(j)}\right)$ is the correlation function and $\theta$ is the parameter. By optimizing the parameters, the correlation between design points can be adjusted. Model of variation function is defined as follows:

$$
R\left(\theta, x^{(i)}, x^{(j)}\right)=\prod_{i=1, j=1}^{n} \operatorname{Ri}\left(\theta, x^{(i)}-x^{(j)}\right)
$$

The smoothness of kriging model, the correlation between nearby data points and quantified observations are controlled by Model of variation function. Model of variation function models commonly used in kriging include the exponential model, exponential Gaussian model, Gaussian model, linear model, spherical model, cubic model, spline function model, and so on. The Model of variation function with Gaussian model can provide a relatively smooth and infinitely differentiable surface, so it is widely used in engineering applications as a variation function. The mathematical expression of Gaussian function is as follows:

$$
R\left(x^{(i)}, x^{(j)}\right)=\exp \left[-\sum_{k=1}^{n} \theta_{k}\left|x_{k}^{(i)}-x_{k}^{(j)}\right|^{2}\right]
$$

Here, $\theta_{k}$ is the correlation parameter, which is the parameter (constant) of the kernel function in the $K$ direction of the sample point. Its main function is to coordinate the flexibility of the variation function in the calculation, and it is an important parameter for the kriging method to establish the approximation model; $x_{k}^{(i)}$ is the coordinate of $x^{(i)}$ in the $K$ direction.

The Gaussian function is chosen as a variation function of the kriging model, its parameters are optimized, and the Root Mean Square Error (RMSE) of the model is taken as an optimization objective function.

By improving the quantum particle swarm optimization algorithm, the parameters of the Gaussian vario-gram function are optimized to improve the fitting accuracy of the kriging method. The accuracy index of the kriging approximation model optimized by the improved QPSO algorithm is shown in Table 4.

Table 4 Precision index of the kriging approximate model before and after the improvement

\begin{tabular}{|c|c|c|c|}
\hline Method & $\mathrm{R}^{2}$ & RMSE & RMAE \\
\hline Kriging & 0.9950 & 3.2887 & 0.2843 \\
\hline Improved Kriging Method & 0.9970 & 2.5097 & 0.1836 \\
\hline
\end{tabular}

According to the above table, the accuracy of the improved evaluation surrogate model has been improved by three indexes: root mean square error (RMSE), R-Square and relative mean absolute error (RMAE); the improved accuracy further improves the establishment of the kriging model. The accuracy of the surrogate model considering the input parameters (pressure angle, tooth width, modulus, tooth line radius, and moment) and the output (contact 
stress) of hyperbolic circular arc tooth-line cylindrical gears lays a foundation for the subsequent analysis. The optimized residual, prediction results, and graph are shown in Figures 5-7.

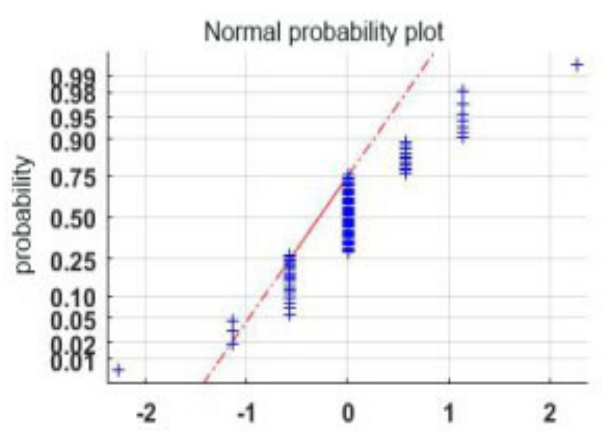

(a) Residual $\quad \times 10^{-13}$

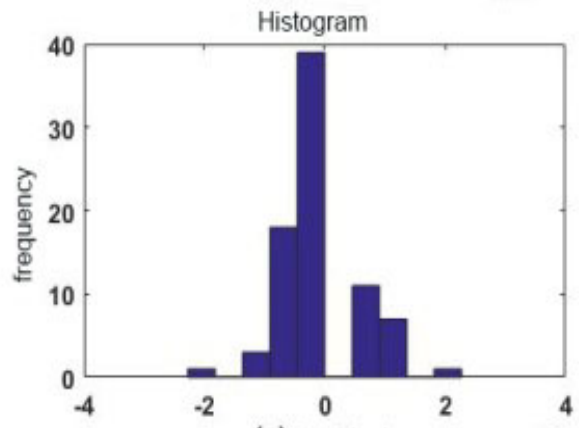

(c) Residual

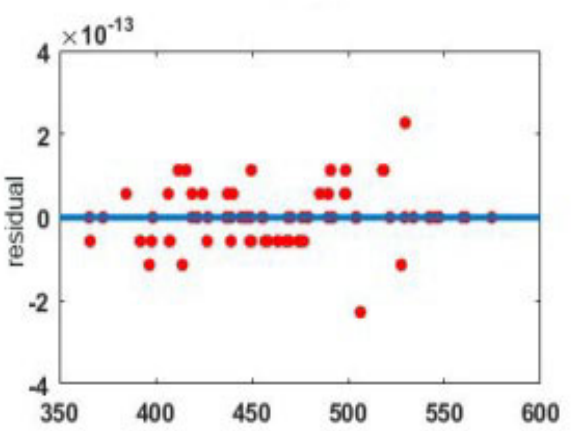

(b) Fitting values

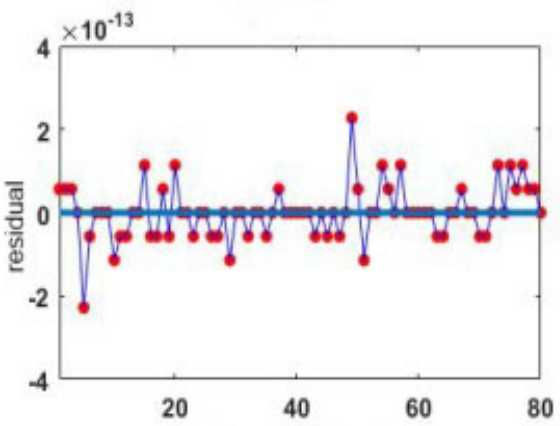

(d) Sequence of observations

Fig. 5 Training set residual diagram based on the AQPSO-kriging surrogate model

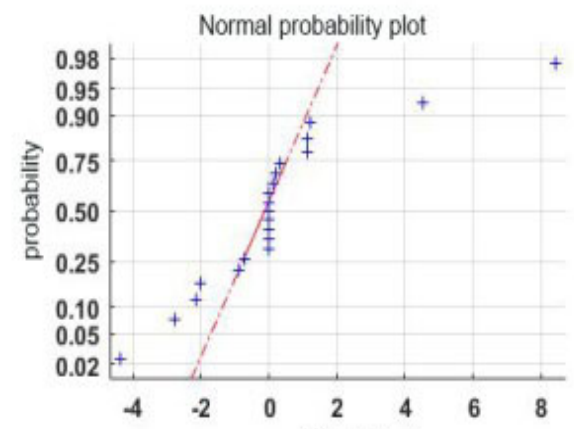

(a) Residual

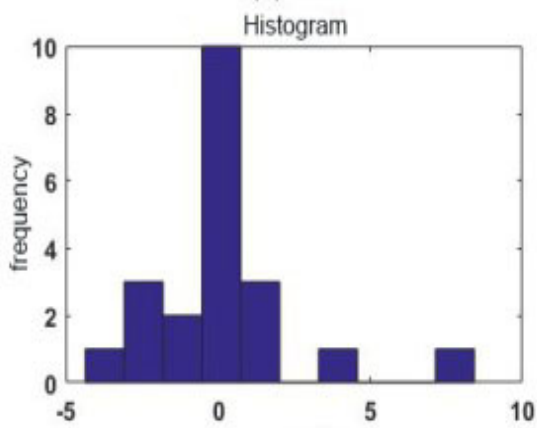

(c) Residual

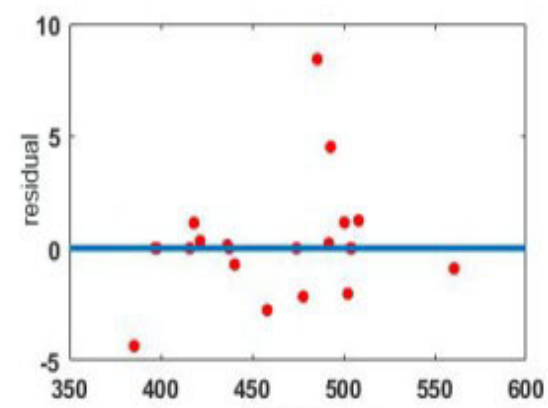

(b) Fitting values

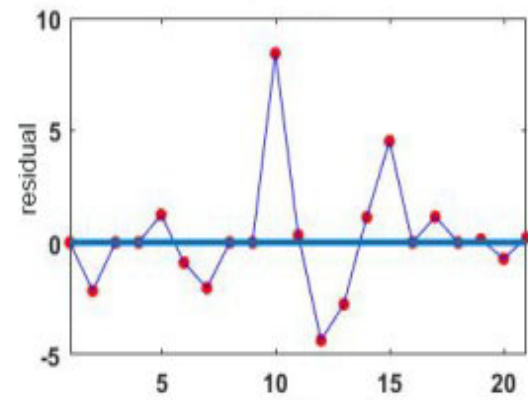

(d) Sequence of observations

Fig. 6 Test set residual diagram based on the AQPSO-kriging surrogate model 
Q. Zhang, G. Wen,

L. Luo, R. Tang

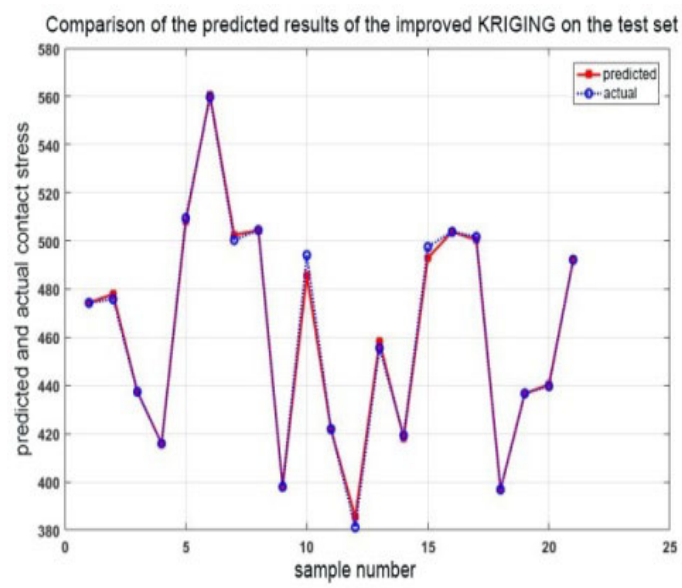

(a) Prediction results
Contact Stress Prediction Model for Variable Hyperbolic Circular Arc Gear Based on the Optimized Kriging-Response Surface Model

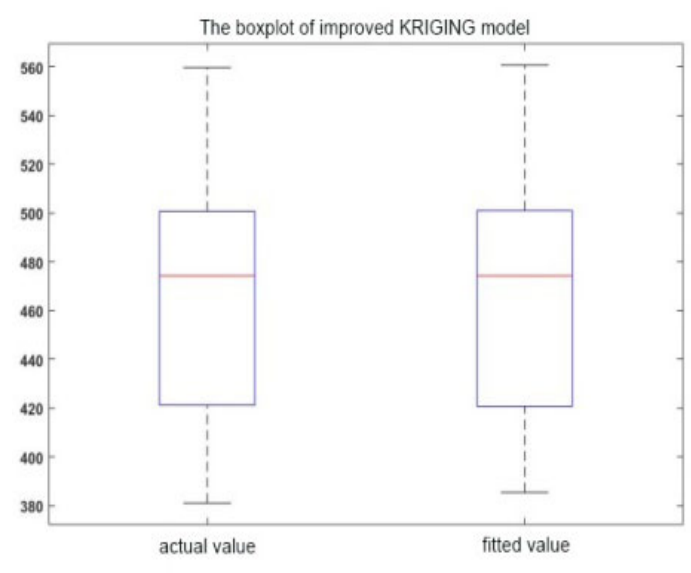

(b) Box diagram

Fig. 7 Prediction results and a box diagram based on the AQPSO-kriging surrogate model

Kriging is used to establish a surrogate model for the relationship between the input parameters (pressure angle, tooth width, modulus, tooth radius, and moment) and the output (contact stress) of VHCAG; the order of magnitudes of error of the surrogate model is $2 \times 10^{-13}$. Only 12 samples from the prediction set can be reproduced well, and they are measured in 9 cases. Although the sample cannot be reproduced well, only one sample has a maximum error of about 13MPA, and the difference between the predicted value and the actual value of eight samples is less than 5MPA. The AQPSO-kriging method is used to establish a surrogate model for the relationship between the input parameters (pressure angle, tooth width, modulus, tooth radius, moment) and the output (contact stress) of the hyperbolic circular arc tooth-line cylindrical gear. The error magnitude is $1 \times 10^{-13}$; however, only 10 samples of the predicted set can be well replicated. At present, although the samples cannot be reproduced well in 12 test sets, only 3 samples have errors greater than 4MPA, and the maximum value is about 8MPA. The difference between the predicted value and the actual value of 9 samples is about3MPA, which effectively improves the accuracy of the model.

\section{Research on the AQPSO-kriging-RSM surrogate model}

A surrogate model of gear design parameters and response values is established based on the AQPSO-kriging method, but the kriging surrogate model cannot directly provide the explicit expression of surrogate model function between input and output. The output of its display expression is realized by conversion, and the explicit expression with terms is obtained, but it cannot be obtained directly. The mathematical expression in polynomial form is quite complex, which is not the subsequent programming of reliability calculation and optimization design. In order to simplify the model, an AQPSO-kriging approximation model based on the gear design parameters and response values is proposed, and polynomial response surface is used to fit the approximation model. The polynomial approximation model between the gear design parameters and response values is obtained. The AQPSO-kriging provides data samples for establishing a response surface model in order to obtain more data. For accurate fitting results, the complete quadratic response surface is selected and the design variables are selected from 4000 sample points between the upper and lower bounds of the design by using the optimized Latin hypercube experimental design method for complete quadratic fitting. The mapping relationship between parameters is established. The optimal Latin square sampling method is used in Isight to sample the upper and lower bounds of variables. Based on the kriging 
approximation model of gear design parameters and response values, the response values of the analysed samples of design variables are calculated, and a binomial response surface model is established. The correlation between design variables and output responses, and the effect of design variables on output responses in the established surrogate model are also discussed. The analysis is carried out. The fitted residual graphical representation is shown in Figure. 8 As can be seen from the figure, the training sample can be reproduced by the response surface method, and the absolute error is less than 2MPA.
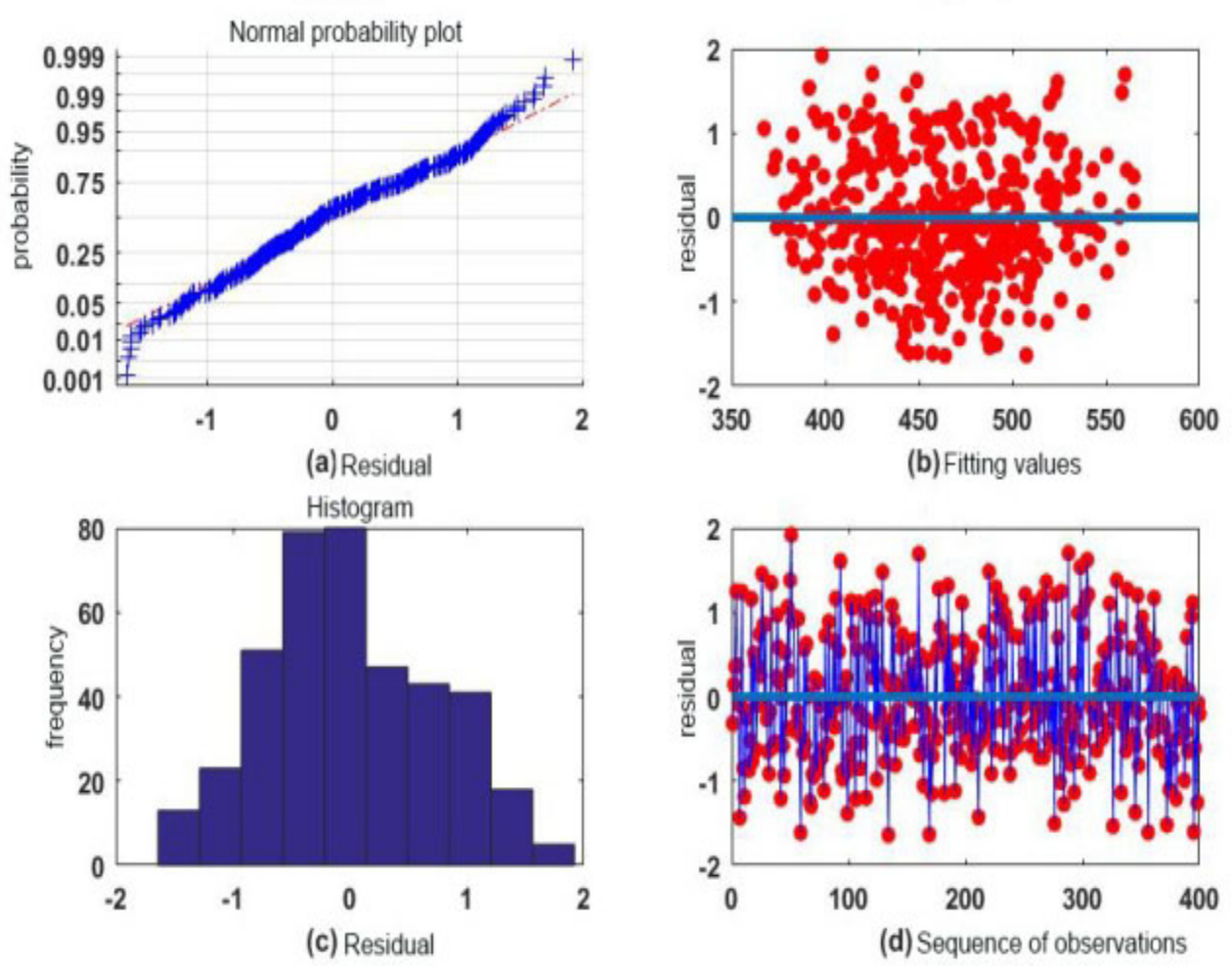

Fig. 8 Residual chart fitted by the drive wheel

A binomial response surface model is established by fitting. The coefficients of the response surface are shown in Equation 16.

$$
\begin{aligned}
y= & 892.858100838364-48.4239995276189 x_{1}-129.022376574498 x_{2} \\
& +27.1928130560591 x_{3}-2.38910303011489 x_{4}+3.19306043457358 x_{5} \\
& +0.842196301494258 x_{1}^{2}+7.03292929829766 x_{2}^{2}-0.35135945763997 x_{3}^{2} \\
& +0.00193576482488664 x_{4}^{2}-0.0023796286525423 x_{5}^{2}+0.575348586969959 x_{1} x_{2} \\
& +0.117854563490473 x_{1} x_{3}+0.0186614852448023 x_{1} x_{4}-0.0338134740010336 x_{1} x_{5} \\
& -0.362050425259949 x_{2} x_{3}+0.137695997619272 x_{2} x_{4}-0.261469117268301 x_{2} x_{5} \\
& -0.000518333663425921 x_{3} x_{4}+0.000487903474894235 x_{3} x_{5} \\
& -0.000136289867271496 x_{4} x_{5}
\end{aligned}
$$

The influence of the parameters on the response surface parameters is analysed in detail. Figure 9 shows the output distribution of the fitted response surface, and Fig. 10 shows the significant degree of the influence of each variable and its group on the contact stress. Red is the negative effect, while blue is the positive one. 
Q. Zhang, G. Wen,

L. Luo, R. Tang

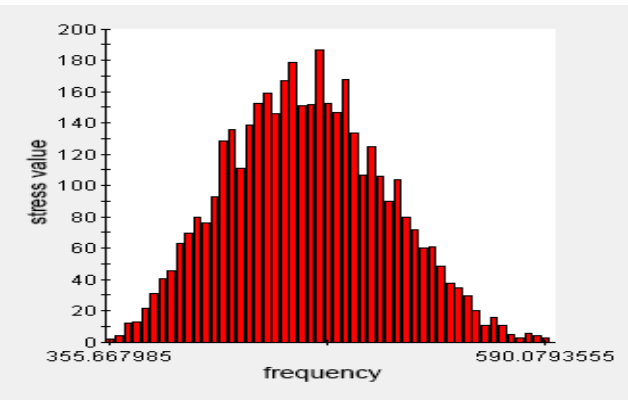

Fig. 9 Response surface output distribution
Contact Stress Prediction Model for Variable Hyperbolic Circular Arc Gear Based on the Optimized Kriging-Response Surface Model

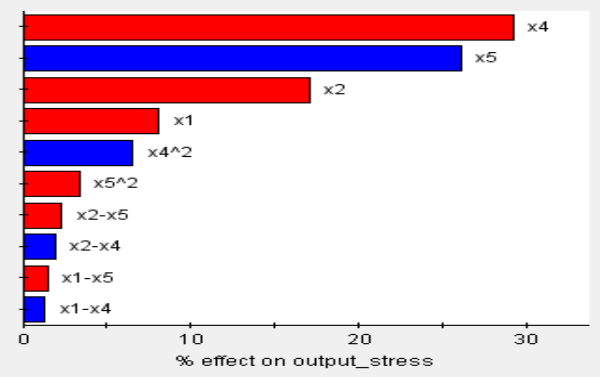

Fig. 10 The effect of design variables on output

According to Figure 9, the output (contact stress) of response surface is normal distribution. In Fig. 10, one can see that the influence of pressure angle, modulus, moment, and tooth line radius on the contact stress increases gradually, and that the increase in the moment is proportional to the increase in stress. The influence of tooth width on the contact stress is not significant and can be ignored, so it can be regarded as a fixed value in the subsequent analysis and design.

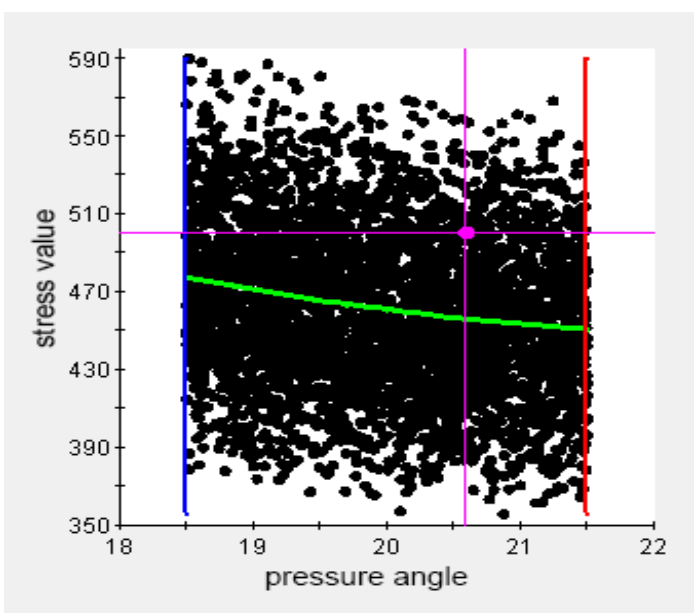

(a) Pressure angle-contact stress

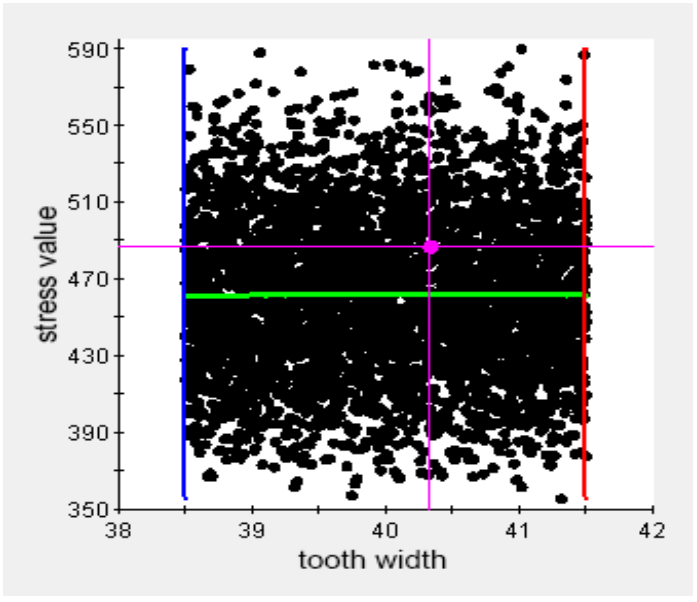

(c) Tooth width-contact stress

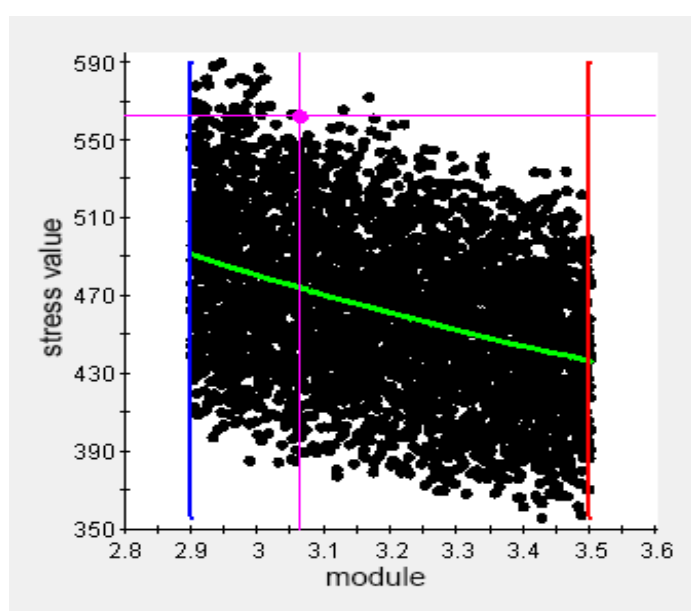

(b) Modulus-contact stress

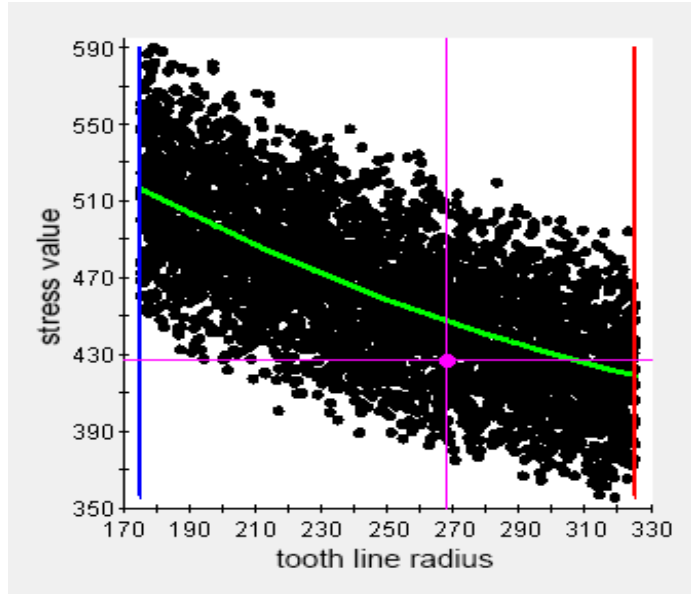

(d) Tooth line radius-contact stress 


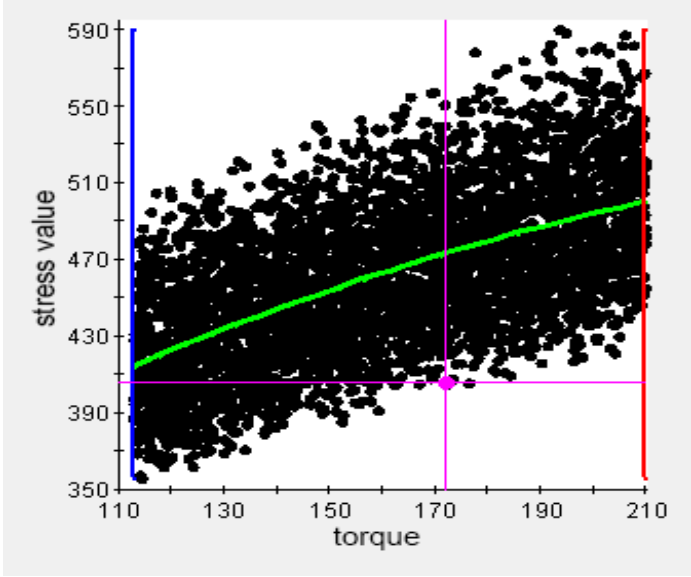

(e) Torque-contact stress

Fig. 11 Design variables and contact stress scatter plots

Figure 11 (a) - 11 (e) show scatter plots of the correlation between the design variables and the output (contact stress). The scatter plots show the distribution of contact stress caused by the change in independent variables, and the solid line (green line) in each plot shows the trend of the change in the contact stress corresponding to the change in independent variables. That is to say, they show the correlation between the change in contact stress and the change in design variables. If the solid line (green line) falls, the contact stress is negatively correlated with the change in design variables; when the solid line (green line) rises, the contact stress is positively correlated with the change in design variables. Thus, one can see that the influence of tooth width on contact stress can hardly be neglected. Secondly, the influence of pressure angle, modulus, radius and moment increases in turn; however, the influence of moment is a positive correlation, while the other three are negative correlations, and the tooth width is basically an irrelevant parameter. The relationship between design variables and contact stress was established, and the correct results were obtained, which can be used for the subsequent reliability analysis and robust optimization design.

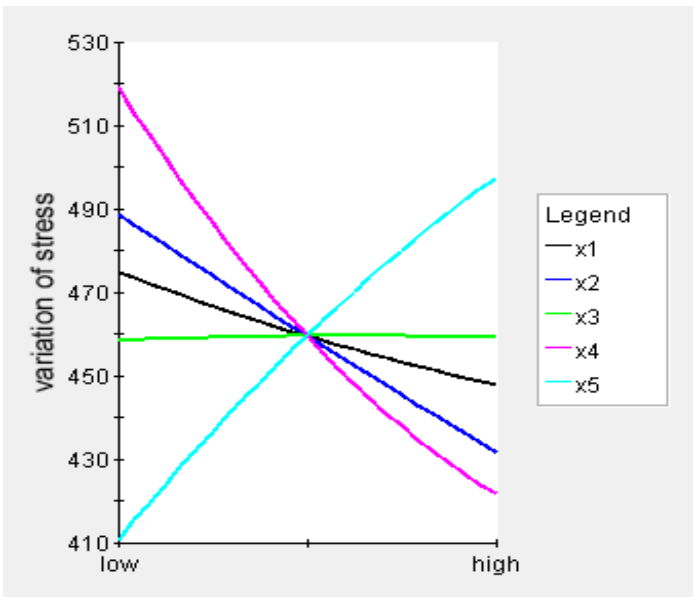

(a) Main effect diagram of design variables

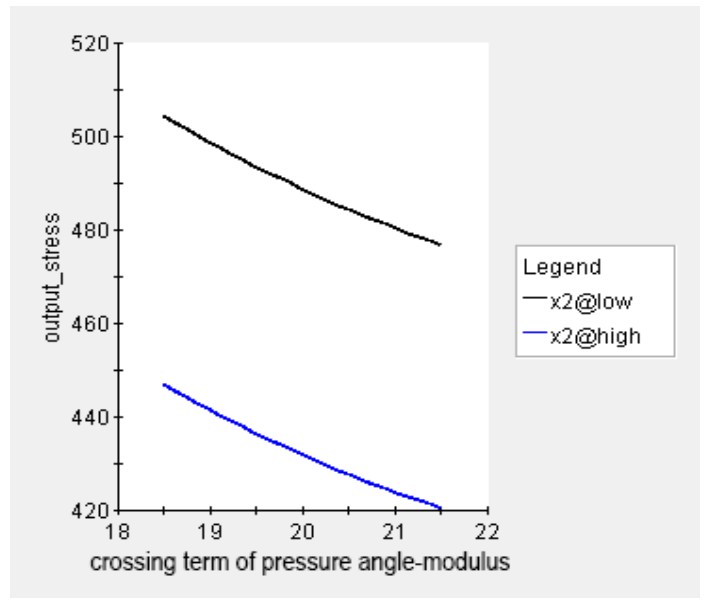

(b) Pressure angle-modulus interaction 
Q. Zhang, G. Wen,

L. Luo, R. Tang

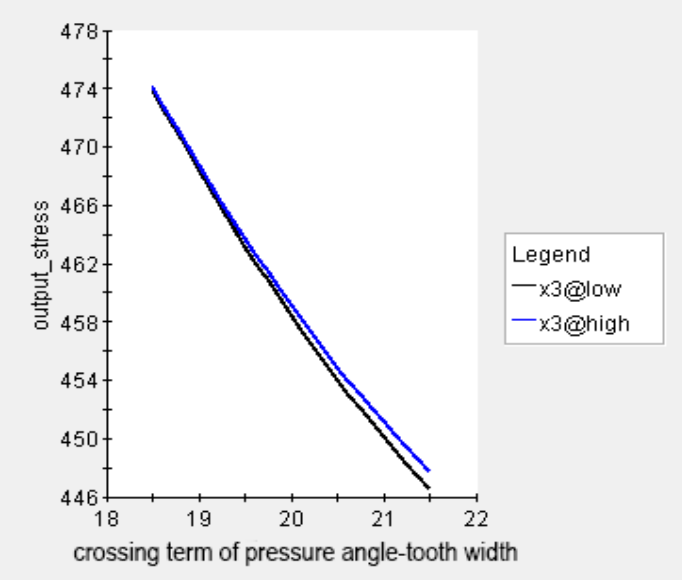

(c) Pressure angle-tooth width interaction
Contact Stress Prediction Model for Variable

Hyperbolic Circular Arc Gear Based on the

Optimized Kriging-Response Surface Model

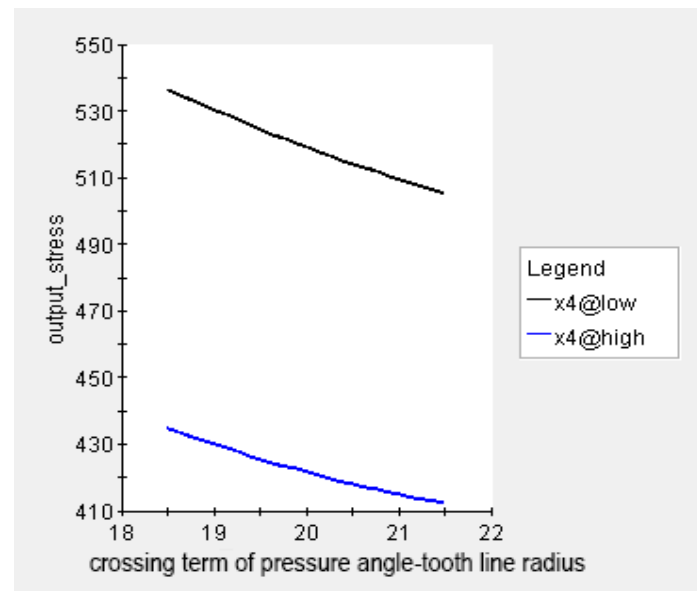

(d) Pressure angle-tooth radius interaction

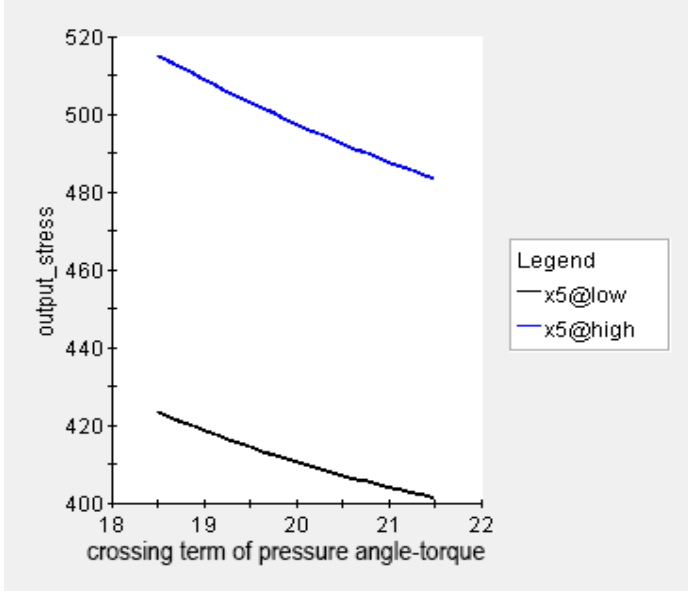

(e) Pressure angle-torque interaction

Fig. 12 The main and interaction diagrams

From Fig. 12 (a), one can see that five design variables, i.e. pressure angle, modulus, tooth width, moment, and tooth line radius, have different effects on the contact stress of gears. Among them, the influence degree of tooth width can be neglected; the degree of influence of tooth line radius, moment, modulus, and pressure angle increases in an ascending order. The influence of pressure angle, modulus, tooth width and tooth line radius on the contact stress is negatively correlated and the moment is correlated. Figure 12 (b) - (e) show the degree of influence of design variables on the response in the case of two-to-two interaction (shown by the pressure angle as an example). If parallel is not interactive, two lines are not parallel or intersected, then the interaction is indicated.

\section{Conclusion}

In order to study the influence of design parameters (pressure angle, tooth width, tooth line radius, modulus, and moment) on the contact stress of variable hyperbolic circular arc gear (VHCAG), and to obtain the best manufacturing parameters, a hybrid kriging-response surface model surrogate model with an adaptive quantum particle swarm optimization (QPSO) algorithm was proposed to establish a model for the expression prediction of the relation between design parameters and contact stress. An intelligent quantum particle swarm optimization algorithm based on adaptive weight and natural selection was proposed to 
optimize the parameters of Gaussian variation function of the kriging surrogate model to improve its fitting accuracy; the global search ability of quantum particles was improved, and the accuracy and stability of the algorithm are improved by adjusting the weight of quantum particles and by optimizing the elimination iteration process.

An intelligent quantum particle swarm optimization algorithm based on adaptive weight and natural selection was proposed. The accuracy and stability of the quantum particle swarm optimization algorithm were improved.

An improved hybrid kriging-response surface model surrogate model with a quantum particle swarm optimization algorithm based on adaptive weight and natural selection was proposed. The quantum particle swarm optimization algorithm based on adaptive weight and natural selection was used to optimize the parameters of the Gaussian variation function of the kriging surrogate model, which improves the fitting accuracy of the kriging surrogate model. The model of expression prediction for the relation between design parameters and contact stress was obtained.

The significant degree of the influence of pressure angle, tooth width, tooth line radius, modulus, moment and their interactions on contact stress is analysed. The results show that the influence of tooth width on contact stress can hardly be neglected, and that the influence of pressure angle, modulus, radius and moment increases in turn.

\section{Acknowledgement}

This project is supported by the National Natural Science Foundation of China (No. 51375320), by the Science and Technology Research Program of Sichuan Province (No. 2018JY0420), and by the Seed Fund of University Science Park of Panzhihua city (No. university science park 2019-25).

\section{REFERENCES}

[1] Gosselin C J, Cloutier L. Generating space for parabolic motion error spiral bevel gears cut by the Gleason method [J]. American Society of Mechanical Engineers, Design Engineering Division (Publication) DE, Advancing Power Transmission into the 21st Century. 1992, 43(1): 229-235. https://doi.org/10.1115/1.2919215

[2] Litvin F L. Synthesis of spiral bevel gears [J]. Gear Technology. 1991, 8(2): 33-35.

[3] Sun Zhijun. Basic Theory Research on Cylindrical Gear Transmission with Variable Hyperbolic Arc Tooth Line [D]. Chengdu: Sichuan University, 2016.

[4] Zhang X, Xie Y, Tan X. DESIGN, MESHING CHARACTERISTICS AND STRESS ANALYSIS OF CYLINDRICAL GEARS WITH CURVILINEAR TOOTH PROFILE[J]. TRANSACTIONS OF FAMENA. 2016, 40(1): 27-44.

[5] Z Ma, X Wang. Research on a Highly Durable and Heavy Duty Gearing [J]. Journal of Xi'an Jiaotong Uni- versity. 2002(03): 282-286.

[6] Lin Ziguang. Study on Pump with Curved Tooth Cylindrical Gear[J]. Mechanical Engineer. 2001(10): 18-20.

[7] Xiang Guoqi, Yin Guofu. Robust Optimization Based on Support Vector Machine and Particle Swarm Algorithm [J]. Machine Design and Research. 2016(06): 6-9.

[8] Cremanns K, Roos D, Penkner A, et al. Robust design optimization of a steam turbine labyrinth seal based on surrogate models[C]. Oslo, Norway: American Society of Mechanical Engineers (ASME), 2018. https://doi.org/10.1115/gt2018-75262

[9] Wu J J, Wan L Q, Nie P F. Kriging surrogate model for compliant mechanism Six Sigma robust optimization design[C]. Singapore, Singapore: IEEE Computer Society, 2016. https://doi.org/10.1109/ieem.2015.7385873 
[10] Wang Jie. RESEARCH ON TOLERANCE ROBUST OPTIMIZATION OF SHEET METAL FORMING BASED ON THE IMPROVED RESPOINSE SIRFACE MODEL [D]. Southwest Jiaotong University, 2014.

[11] Dan Y, Hongbing J, Yongchan G. A robust D-S fusion algorithm for multi-target multi-sensor with higher reliability[J]. Information Fusion. 2019, 47: 32-44. https://doi.org/10.1016/j.inffus.2018.06.009

[12] Mentgomery Douglas C. Design and Analysis of Experiments: 6 edition [M]. Wiley, 1998.

[13] TONG Cao, SUN Zhili, CHAI Xiaodong, WANG Jian. Gear Contact Fatigue Reliability Based on Response Surface and MCMC [J]. Journal of Northeastern University (Natural Science). 2016(04): 532-537.

Submitted: $\quad 01.12 .2019$

Accepted: $\quad 21.9 .2020$

\section{Zhang Qi}

Chengdu Industrial Vocational Technical College, Chengdu 610218, China

Panzhihua University, Panzhihua 617000,

China

Wen Guang*

57438701@qq.com

Chengdu Industrial Vocational Technical

College, Chengdu 610218, China

Luo Lan

Sichuan University, Chengdu 610065, China

Tang Rui

Civil Aviation Flight University of China

No. 46, Section 4, Nanchang Road, Guanghan

City, Sichuan Province, China 